\title{
El Portal del Patrimonio Histórico de Andalucía
}

Carmen Ladrón de Guevara Sánchez Susana Limón Rodríguez Isabel Ortega Vaquero

Centro de Documentación del IAPH

\section{Disquisiciones varias y pasos iniciales}

No sé si fue un sueño, una ilusión o una incógnita en el infinito, pero imaginar o siquiera soñar que un día pudiéramos "ver" la Gioconda en nuestra mesa de trabajo, tener acceso a múltiples direcciones, sin consultar las páginas amarillas, oír música o.... no, quizás nunca lo hubiéramos adivinado ni siquiera deseado. Pero hoy, eso y mucho más, es posible.

Si acaso la posibilidad fuera la de sentirte espectador privilegiado, pero es todavía más. Eres copartícipe de todo un mundo comunicativo en el que entras, creas y contemplas cómo tu mensaje interesa, llega y es apreciado.

No, no era posible escapar a la tentación, así que pensamos participar en el festín y hacernos conocer por un gran potencial de público.

En noviembre de 1995 nos planteamos el reto de la incorporación del IAPH a Internet con el deseo múltiple de: comunicar nuestro proyecto de Institución, presentar el patrimonio histórico y servirnos de la red como vía de acceso a un mundo de posibilidades informativas en permanente descubrimiento.

Nuestra trayectoria ya merecía la pena contarla. Las diversas áreas del Instituto estaban embarcadas en proyectos culturales cuya experiencia, resultados, metodología e innovación queríamos transmitir.

No hay nada más favorable para la consecución de un objetivo que embarcar en el mismo a un grupo de personas con el mismo nivel de ilusión. Esa fue nuestra primera misión. Fácil, contamos desde el principio con el apoyo estratégico de la dirección y con la complicidad de todas las áreas que conjuntamente íbamos a impulsar el proyecto.

Era unánime el convencimiento de su potencialidad como escaparate, foro y espacio interactivo de comunicación con otras personas, asimismo interesadas en todo este mundo de proyección de nuestra cultura.
El Web permite integrar en un entorno común diferentes tipos de información, en diferentes formatos; es capaz de transmitir información de forma masiva; posibilita la rapidez de la consulta; localiza informaciones especializadas, con ausencia de horarios y de impedimentos de localización geográfica...

En fin, todo un capital de posibilidades que desde el principio nos obligó a disciplinarnos en el ritmo de consecución de nuestros objetivos y en el control del rigor de la información a transmitir.

La promoción de nuestra imagen corporativa con la difusión de sus actividades, servicios, recursos informativos y la presentación del patrimonio histórico de Andalucía son los dos pilares sobre los que pivota nuestro mensaje en la red.

El primero porque queríamos transmitir nuestra experiencia y dar a conocer sus resultados y el segundo, de mayor envergadura, porque creemos que es necesario difundir nuestra riqueza cultural a aquellos interesados en acercarse a ella.

Además queríamos crear un punto de encuentro con el usuario. $Y$ es que Internet no es un medio de sentido único, opera en las dos direcciones y permite entrar en contacto con los usuarios, fomentando un sentimiento de participación que no existe en productos convencionales.

Decididos a acometer la empresa nos pusimos manos a la obra de diseñar y desarrollar un Servidor Web propio.

Los hitos principales en su proyección temporal desde sus inicios hasta ahora vienen marcados por los siguientes pasos:

Nuestra conexión a la red RICA (Red Informática Científica de Andalucía) a través del CICA (Centro Informático Cientííco de Andalucía) durante 1996.

El desarrollo de la información institucional estructurada según las áreas organizativas y acorde con las principales materias informativas de las mismas, así como el enlace a otros servidores web de interés, tanto especializados como institucionales y a buscadores generales de información.

Se potencia la interactividad con nuestros usuarios poniendo a su disposición distintos formularios, como el de suscripciones al Boletín PH, el de inscripción a los cursos de Formación, y el de Demandas Puntuales de Información. 
Se inicia una primera información básica del patrimonio histórico de Andalucía con un impulso al botón "Patrimonio Histórico Andaluz", mostrando un primer estudio sobre Granada capital, los Bienes Patrimonio de la Humanidad, los Bienes de Interés Cultural (B.I.C.) y Rutas o Itinerarios por la ciudad; completado posteriormente con una selección de 54 edificios representativos de todas las provincias del "Registro Andaluz de Patrimonio Arquitectónico Siglo XX. Desarrollo del Registro Docomomo lbérico (1925-1965).

Se crea un botón denominado Productos Culturales, donde se incluyen Intervenciones Experimentales del IAPH y el Itinerario cultural de Velázquez, coincidiendo con la celebración del IV Centenario de su nacimiento en Octubre de 1999.

Se implanta un botón destinado a informar a los usuarios de las Novedades en el servidor web.

Se inicia el servicio de acceso a las bases de datos de patrimonio histórico con la información del patrimonio arqueológico de datARQUEOS (Base de Datos del Sistema de Información del Patrimonio Arqueológico).

Todo acompañado de un proceso permanente de revisión y actualización de la información existente manteniendo al día especialmente la oferta formativa del $\mathrm{IAPH}$ y la de las publicaciones del mismo.

\section{Una etapa a afrontar}

Después de este transcurrir nos hemos planteado el comienzo de un proceso de salida permanente de la información del patrimonio histórico en la red, como signo de transparencia, necesidad de comunicación y expresión de nuestras inquietudes en el deseo y objetivo de acercamiento del patrimonio a todos.

Por ello nada mejor que aprovechar ese término acuñado para su uso en la red como es el de PORTAL. Suena a tránsito y también a permanencia; lugar de encuentro donde podrán tener cabida temáticas, que hagan de columna vertebral, junto a los avatares cotidianos de la contingencia de nuestro patrimonio.

Adentrarnos en el conocimiento de lo que supone este concepto y posteriormente avanzar algo de su contenido proyectado es lo que nos proponemos a continuación.

\section{¿A qué llamamos "Portal" en Internet?}

En los últimos tiempos la palabra "portal" está de moda por el éxito que estos "concentradores de información" están obteniendo en la red en conseguir y retener usuarios. Un portal es en sí mismo un inmenso "montaje" para enganchar a los internautas; una puerta a través de la cual te diriges hacia otros destinos.

El posicionamiento de algunas importantes empresas españolas en los medios de comunicación ha contribuido a hacer de todos conocido este término, que supone en su concepto y planteamiento una nueva era en el sistema de navegación por Internet. Los expertos auguran que los portales tendrán una importante presencia en Internet durante bastante tiempo, y que son el intermedio entre la Internet actual y la televisión interactiva.

El principal objetivo de todos ellos es no luchar por conseguir el mayor número de visitantes, sino conseguir que estos estén el máximo tiempo posible navegando dentro de las fronteras del propio portal. Dentro de estos grandes "concentradores de información", se incluyen portales, buscadores y otros, que son los mayores concentradores de tráfico en la red.

Entre los portales, cabe distinguir dos categorías principales: portales genéricos u horizontales y portales temáticos o verticales.

Los primeros son los también llamados generalistas, páginas web que ofrecen información general y están enfocadas a todos los públicos, y que se basan en un gran espectro de servicios y contenidos sin profundizar en ninguna materia concreta y que se han convertido en los líderes dentro de la red española. Entre ellos detacan Terra (Telefónica), eresMas (Retevisión), Ya.com (Jazztel), Wanadoo (Uni2) y Navegalia (Airtel). Todo ello, sin contar con la potente presencia de modelos americanos como AOL, Amazon, Yahoo, Excite o Geocities, que son los grandes triunfadores por el incremento de usuarios que han obtenido en el último año. Generalmente, estos portales o comunidades virtuales en mayor grado de desarrollo son las que pertenecen al grupo del ocio, ubicadas especialmente en Estados Unidos, cuyo objetivo principal es ofrecer sitio gratis para la ubicación de páginas personales, servicio gratuito de correo electrónico o suministrar productos a través de tiendas virtuales.

El problema que presentan estos grandes portales es que todos están basados en un mismo bloque de servicios: chats, mensajes a teléfonos móviles, noticias, promociones y ofertas de todo tipo... Incluso el diseño de los contenidos de cada uno de ellos ofrece grandes similitudes. Por ello es necesario que los portales horizontales planteen servicios que hagan más accesible la búsqueda de información por la red. Que cada vez los contenidos especializados y locales tengan más relevancia.

La tendencia actual es por tanto el ir reduciendo los portales genéricos evolucionando con la red hacia nuevas fórmulas: portales temáticos o verticales, portales corporativos, portales personales o ciudades digitales. La especialización y la personalización se imponen, dando lugar a la nueva era de portales temáticos más próximos a las necesidades concretas 
de las diferentes comunidades y grupos de usuarios. Concebir un nuevo espacio adecuado para que un profesional pueda desarrollarse plenamente dentro de un contexto científico y estar continuamente actualizado en sus temas de interés, al margen del lugar geográfico. En definitiva lo que interesa es hacer Internet asequible, útil y cercano para un número de usuarios cada vez mayor.

\section{Portales Temáticos}

En los tiempos más recientes, después de la explosión informativa del Web, asistimos a la irrupción de los Portales Temáticos de información en Internet como expresión de Comunidades Virtuales especializadas (CV). El uso creciente de Internet para la difusión de información altamente especializada y como foro de reunión de especialistas en diferentes temáticas, ha propiciado la creación de las llamadas comunidades virtuales de usuarios. Estas estructuras tienen como objetivo englobar el mayor número de recursos informativos especializados de interés, y permitir el acceso a ellos, ya sea a través de suscripción o gratuitamente.

A diferencia de los portales genéricos que están dirigidos a internautas noveles, los portales temáticos van dirigidos a los que buscan información más concreta imposible de encontrar en un genérico, es decir, a comunidades de usuarios con intereses afines. Los portales temáticos deben tener la capacidad de donar servicios a un segmento más concreto de usuarios y que es difícil de imitar. Estos servicios conllevan una serie de aportaciones de interés, ya que reúnen en un solo sitio web una gran cantidad de información especializada, los contenidos presentados son generalmente de alta calidad, y la información y los servicios ofrecidos se actualizan con gran dinamismo. Constituyen, por tanto, importantes "plataformas informativas" en auge construidas en base a una filosofía de segmentación de contenidos dentro de una temática concreta.

Una de las claves de los portales temáticos está basada en el concepto de Integración de información especializada, que facilita el acceso fácil e inmediato a productos y servicios de información temáticos para ser usados por una comunidad definida de usuarios. Idealmente suministran una visión integrada de toda la información que contiene, propia y externa, sin importar su procedencia, forma, formato, soporte, etc.

Proporcionar un acceso unificado e integrado a recursos especializados distribuidos y diferentes de alta calidad, mejora y expande los servicios a los usuarios en beneficio de una comunidad particular, cuyos miembros se encuentran completamente dispersos y acceden a la información a través de la red. Un portal temático es un Foro de reunión de especialistas, cuya filosofía principal es que cualquier miembro perteneciente a la comunidad virtual debería aportar conoci- mientos, ofrecer nueva información, y no limitarse al mero hecho de obtener información.

Respecto a los Contenidos de alta calidad, los portales ofrecen al usuario una serie de productos y servicios informativos, que podemos agrupar en diferentes apartados. Por un lado, aquéllos aspectos técnicos de acceso a la información que posibilitan la navegación y la recuperación de información, dentro y fuera del portal: índice temático, mapa del web, buscador interno, buscadores externos, y elementos multimedia.

Por otro lado, destaca la presencia de documentación referencial, que aglutina productos como bases de datos propias y externas, catálogos de bibliotecas y de librerías, directorios de recursos web, directorios de eventos, cursos, congresos, concursos, de organismos, instituciones públicas, empresas, asociaciones profesionales, centros de investigación, de profesionales, y novedades bibliográficas.

Asimismo, encontramos otros productos que ofrecen documentación de contenido, con acceso al texto completo de publicaciones propias y externas, textos electrónicos de informes, artículos de revistas, ponencias, comunicaciones..., normativa, páginas temáticas, glosarios y diccionarios, noticias propias y externas.

Por último, con una presencia aún modesta, destacan los servicios a los usuarios, tales como alertas técnicas con perfil o vía e-mail, asesorías técnicas, asesorías legales, foros de discusión, bolsas de trabajo, servicios de correo-e, alojamiento de páginas personales, software, ofertas especiales de compra, y cursos on line.

En general, existe una mayor presencia de la documentación referencial y de los aspectos técnicos de acceso a la información, seguida de la documentación de contenido. Por tanto, la información de carácter referencial sigue siendo muy importante, destacando dentro de la misma el acceso a bases de datos externas, catálogos de libros, directorios web, convocatorias y otros directorios generales, aunque poco a poco se van implantado productos de información final, desde publicaciones y textos electrónicos a noticias y páginas temáticas.

Sin embargo, un aspecto escasamente representado son los servicios. Además, existe un mayor desarrollo de servicios tradicionales de uso general (bases de datos, directorios, foros de discusión, etc.) en contraposición a la escasa presencia de nuevos servicios orientados a satisfacer las necesidades particulares y especializadas de los usuarios (alertas técnicas, asesorías, cursos on line, etc.).

A continuación destacamos algunos ejemplos de portales existentes en la Red sobre diversas temáticas especializadas, donde se describen los segmentos informativos específicos que contienen y que va dirigidos a una comunidad de profesionales concreta. 


\section{Arquinex: Arquitectos de España. Portal de Arquitectura y Construcción}

Los contenidos que presenta son: Direcciones de la Construcción, Direcciones Web, Buscadores de Información, Concurso de Arquitectura, Boletines Oficiales, Cartografía, Normalización y Legislación, Administración y Revistas; todo ello con la vocación de crear una red ideal de comunicación para el sector de la Arquitectura y la Construcción (administración, colegios profesionales, técnicos, constructores, fabricantes, instaladores, etc.)

www.arquinex.es

\section{Comunidad virtual ICT}

Comunidad tecnológica del Instituto Catalán de Tecnología dividida en varias áreas profesionales. Muestra estudios, trabajos, artículos, empresas, publicaciones y otros recursos on line relacionados con el marketing y el comercio electrónico. Cada una dispone de un foro, una lista de distribución, un chat, un boletín de noticias y gran cantidad de servicios formativos, informativos y lúdicos.

www.ictnet.es/esp/comunidades/openmkt

\section{Ecoplus: Primer Portal de Ecología, Medio Ambiente y Tecnología}

Presenta Foros y Grupos de Discusión relacionados con el tema, así como Canales Temáticos: noticias, legislación, documentación, Páginas Verdes, etc.; Servicios: Libros especializados, Tablón de Anuncios, Correo Noticioso, Hemeroteca, entre otros; Referencia: QQué es Ecoplus?, FAQ, Prensa, etc. www.ecoplus.org/default.php3

El Portal del Patrimonio Histórico se concibe como un servicio digital integrado de la información patrimonial desarrollada por el Sistema de Información del Patrimonio Histórico de Andalucía (SIPHA), así como de otros recursos informativos que se encuentren fuera de los límites del propio Sistema. Pretende suministrar una visión integrada de toda la información que contiene el Sistema, sin importar su forma o formato, lo impreso o lo digital, 10 real o lo virtual..., proporcionando un acceso unificado, fácil y rápido a recursos distribuidos y diferentes a una comunidad especializada, y a la vez diversificada de usuarios, resolviendo las barreras geográficas y temporales, y multiplicando el efecto de la difusión y divulgación de servicios y productos de información de ámbito patrimonial.

\section{Un compromiso actual con el futuro: El "Portal" de patrimonio histórico de Andalucía}

Transcurrida una etapa de compromiso y reto con toda una ingente labor por acometer y que afrontamos con la mayor de las ilusiones desde el Centro de documentación y que ha dado unos resultados de los que nos encontramos orgullosos, era casi "natural", en el avance que nos propusimos como premisa permanente, abordar nuevos medios de expresión, difusión y comunicación de la información que en estos años y desde perspectivas innovadoras hemos ido produciendo, sistematizando y poniendo al servicio del público.

El desarrollo del Sistema de información del patrimonio histórico de Andalucía ha apostado, en una primera etapa, por la producción de bases de datos de patrimonio histórico, en la que ha cubierto una gran carencia, avanzando en el entendimiento de que es posible aunar conocimiento y actualización permanente, a través de unas herramientas que recogen el estado de la cuestión casi al día.

En el momento actual apuesta, en un doble sentido, por la distribución de esas herramientas y su implantación en los mecanismos de gestión y por la elaboración de unos productos que den salida a todo ese capital informativo que posee y que está capacitado para incrementar.

Hoy día hay que tener el convencimiento de que el administrado ya no es aquel con el que nos relacionamos a través de unos mecanismos jurídicos, sino que en el momento actual las relaciones hay que entenderlas llevadas al campo del espectador al que queremos mostrar nuestros resultados.

De ahí el haber abordado dos líneas nuevas de presentación de nuestro patrimonio, como la elaboración de productos digitales en Cd-Rom y la información on line a través de la red en la plataforma de nuestro Servidor Web.

La evolución de nuestro servidor web nos lleva a apostar en el momento actual por una información temática que equilibre la doble vertiente, institucional y temática, inicialmente planteada.

Si en las fases iniciales hemos desarrollado una serie de aproximaciones a contenidos temáticos que nos acercaran a un diseño y a un desarrollo más maduro de la información patrimonial, ahora estos productos iniciales nos han llevado a plantearnos una alternativa nueva para presentar dicha información, una nueva forma denominada "Portal".

La apuesta del Centro por la producción de productos digitales y por la difusión telemática de la información del ph, viene además reforzada por el impacto de nuestro servidor web y por el uso de sus contenidos. Según los datos estadísticos de visitas a nuestra página web, nos encontramos con un incremento progresivo 
de accesos (66.000 en octubre 2000) y con una demanda explícita de productos culturales, p.e. Velázquez, Base de datos Arqueos y Docomomo como secciones más visitadas.

Dado que existe una creciente presencia de portales temáticos en la red y una importante demanda de recursos de información de patrimonio histórico basados en las nuevas tecnologías, parece oportuno en este momento plantearnos este modelo de contenidos aplicado al patrimonio histórico.

Se presenta como una importante clave para el desarrollo de la infraestructura de la información patrimonial, para la innovación, para la investigación, y para la transferencia de conocimientos a la sociedad. La creación de una óptima infraestructura informativa favorece el desarrollo sostenido de la producción, acceso y disponibilidad de los contenidos y de los recursos de información del Sistema. Gestionar el cambio y la transición de las formas tradicionales a lo digital y multimedia supone innovar en la metodología de creación y difusión cultural. Para la investigación supone un banco de información al servicio del conocimiento, y para la transferencia implica un acercamiento y transmisión de conocimientos a la sociedad.

De acuerdo con las múltiples posibilidades actualmente presentes en los portales de la red, consideramos que las alternativas de contenidos al portal del patrimonio histórico son diversas y extensas. Los productos informativos y servicios contemplados como relevantes para su inclusión, nos lleva a la consideración de una larga lista de posibles recursos: Canales Temáticos, Tribuna, Bases de datos, Productos Culturales, PH Recomendado, PH Olvidado, Itinerarios Culturales, Directorios de organismos, de profesionales, de eventos, de cursos, etc., Publicaciones, Noticias, Legislación, Servicios de alerta, Motor de búsqueda, Registro usuarios, Buzón, Foros, y un largo etcétera de opciones imposibles de abordar de forma completa y lineal.

Por ello, conscientes de la disponibilidad actual de nuestros recursos y de acuerdo a un faseado priorizado del proyecto, nos planteamos en una primera etapa el desarrollo y disponibilidad de los siguientes contenidos:

\section{Tribuna PHA}

que ofrece una visión global y una valoración de nuestro patrimonio con la finalidad de plantear una reflexión analítica sobre las peculiaridades del pha.

Además de esta información de carácter más estable, se incorporarán de forma paulatina y periódica nuevos temas que por su interés y actualidad se consideren de especial relevancia, con el fin de mantener una tribuna que refleje los principales debates en torno al conocimiento del ph y de los bienes culturales andaluces.

\section{Faq PHA,}

que plantea las preguntas-respuestas más frecuentes sobre el patrimonio, de forma que los usuarios obtengan de forma directa y fácil un conocimiento básico y/o especializado sobre las principales cuestiones que afectan al mismo, agrupadas por temas y niveles de especialización.

\section{Productos Culturales,}

donde se incluirán recursos de producción propia específicos y especializados sobre el pha, mediante la adaptación de productos ya desarrollados y/o la elaboración de nuevos productos para este medio (dossiers temáticos, productos multimedia, etc.). Estos productos irán enfocados hacia un bien cultural concreto, a una tipología patrimonial, a un artista, a una obra, etc., p.e. Ermitas, Velázquez, la Mezquita de Córdoba, etc

\section{Base de datos del pha,}

que facilitará el acceso y recuperación de la información de los bienes culturales andaluces y de las fuentes de información del patrimonio histórico de Andalucía, a través de la consulta de la Base de Datos Integrada del Patrimonio Histórico de Andalucía.

\section{Itinerarios Culturales,}

que se configura como una nueva propuesta para la valoración y disfrute del pha. Con ello, se propone ampliar las nuevas formas de lectura del concepto patrimonio cultural basándose en el punto de vista del disfrute social y uso in situ del patrimonio conservado. Ello se plasmará en un recorrido virtual por el entorno geográfico, la historia, los elementos culturales... de cada una de las rutas temáticas a visitar.

\section{Canales Temáticos,}

que representan una puerta de acceso a los recursos de información relativos a diferentes ámbitos temáticos de interés para el patrimonio histórico. Su objetivo es ofrecer una primera visión general y unificada de los recursos disponibles en la red, así como las noticias y novedades producidas en cada uno de los ámbitos especííicos.

En una primera fase se prevé abrir los canales temáticos relativos a Patrimonio Arquitectónico, Patrimonio Arqueológico, Patrimonio Mueble, Patrimonio Etnológico, Instituciones PH, Conservación y Restauración, Nuevas Tecnologías de la Información.

Cada uno de los canales contendrá los recursos de información presentes en Internet, a través de la recopilación, selección y descripción de los webs de interés (organismos, publicaciones, bases de datos, documentos a texto completo, productos, etc.), así como una selección de las noticias y novedades más destacadas en cada uno de los ámbitos. 
Lo + web,

donde se destacará la reseña comentada del web del mes.

\section{Publicaciones ph,}

con acceso a las publicaciones electrónicas editadas de forma interna (Boletín PH, Cuadernos, Dossiers Temáticos ...), a través de la inclusión de los sumarios y de artículos a texto completo.

\section{Agenda ph,}

que incluirá información sobre los cursos de especialización, cursos de posgrado, congresos, jornadas, etc., internos y externos.

\section{Opinión,}

se trata de crear un canal electrónico de comunicación fluido y rápido con los usuarios para recoger las opiniones, sugerencias, comentarios, propuestas sobre determinados temas que se vayan planteando, así como sobre la información disponible en el portal.

Asimismo se dispondrá de una serie de Herramientas de ayuda para dirigir el acceso a la información del portal, consistente en una página de Presentación general y ayuda; un Mapa del portal que represente su organización interna y oriente en su navegación; un
Buscador interno como herramienta de búsqueda de los contenidos incluidos en el portal; y Buscadores externos para la recuperación de recursos disponibles en la Web.

\section{Idea final}

Nuestra propuesta, materializada en el portal temático del pha, trata de construir una superestructura en la que albergar todos los recursos y servicios que de otra manera estarían dispersos en diferentes sitios de la red, y en la que ofrecer productos y servicios no disponibles en ningún otro lugar, no sólo por la cantidad sino por la calidad.

Esto supone un importante paso hacia la transferencia del conocimiento sobre el patrimonio histórico, apoyada en nuevos medios de transmisión que ponen al alcance de todos la especialización de nuestros contenidos y que facilita la interacción e intercambio de conocimientos entre los profesionales.

Por ello, nuestros esfuerzos se encaminan a la creación de productos finales de información que facilitan el acceso a contenidos especializados de alta calidad, propios y externos, a través de una puerta virtual "única" de información del patrimonio histórico andaluz.
CASTILLO VIDAL, J. "Comunidades virtuales: la superación de las listas de distribución como foro de reunión y trabajo de profesionales". En El Profesional de la Información, vol.8, nº 6, junio 1999. Barcelona: Swets \& Zeitlinger Ibérica, 1999, pp. 14-28

CORREDOR, J. "Portales en España: renovarse o morir". En Baquia, agosto 2000

http://www.baquia.com

"Els portals escruten el seu futur". En Tecnología Propera, septiembre 2000

FERNÁNDEZ SÁNCHEZ, E., FERNÁNDEZ MORALES, I. - MALDONADO MARTÍNEZ, Á. "Comunidades virtuales especializadas: Un análisis comparativo de la información y servicios que ofrecen al usuario". En VII Jornadas Españolas de Documentación (Bilbao 19-21 octubre 2000). Bilbao: Universidad del País Vasco, 2000, pp. 383-390
PETERSON, R.L. y FLANIGAN, E.J. "Impacts of the "new" networked communities". En: Procedings of the 1997 Information. Hersey (EUA): Idea group publishing, 1997.

RHEINGOLD, H. "The virtual community: home steading on the electronic frontier". En: Online, marzo 1994, v. 18 , n.2, pp.68-69.

ROMM, C., PLISKIN, N. Y CLARKE, R. "Virtual communities and society: toward and integrative three phase model". En International journal of information management, agosto 1997, v. 17, n.4, pp.261-270

VALLE, A. "Bendito portal". En Actualidad Crónica, 2000.

WILKINSON, J. "The art and craft of creating community". En Digital publishing strategies, octubre 1997, v.2, n.2, p.8 